\title{
Sensitivity of ovarian cancer cells to acetaminophen reveals biological pathways that affect patient survival
}

\author{
STEPHEN H. BUSH ${ }^{1}$, SHARON TOLLIN ${ }^{1}$, DOUGLAS C. MARCHION ${ }^{1}$, YIN XIONG ${ }^{1}$, FOROUGH ABBASI ${ }^{1}$, \\ INGRID J. RAMIREZ ${ }^{1}$, NADIM BOU ZGHEIB ${ }^{1}$, BERNADETTE BOAC ${ }^{1}$, PATRICIA L. JUDSON ${ }^{1}$, \\ HYE SOOK CHON ${ }^{1}$, ROBERT M. WENHAM ${ }^{1}$, SACHIN M. APTE ${ }^{1}$, CHRISTOPHER L. CUBITT ${ }^{2}$, \\ ANDERS E. BERGLUND ${ }^{3}$, LAURA J. HAVRILESKY ${ }^{4}$ and JOHNATHAN M. LANCASTER ${ }^{1}$ \\ ${ }^{1}$ Department of Women's Oncology; ${ }^{2}$ Translational Research Core; ${ }^{3}$ Cancer Informatics Core, H. Lee Moffitt Cancer \\ Center and Research Institute, Tampa, FL 33612; ${ }^{4}$ Duke University Medical Center, Durham, NC 27710, USA
}

Received February 11, 2015; Accepted November 16, 2015

DOI: $10.3892 / \mathrm{mco} .2016 .725$

\begin{abstract}
Experimental and epidemiological data support the potential activity of acetaminophen against ovarian cancer (OVCA). In the present study, we sought to confirm the activity of acetaminophen in OVCA cell lines and to investigate the molecular basis of response. A total of 16 OVCA cell lines underwent pretreatment (baseline) genome-wide expression measurements and were then treated with and analyzed for acetaminophen sensitivity. Pearson's correlation analysis was performed to identify genes that were associated with OVCA acetaminophen response. The identified genes were subjected to pathway analysis, and the expression of each represented pathway was summarized using principal component analysis. OVCA acetaminophen response pathways were analyzed in 4 external clinico-genomic datasets from 820 women for associations with overall survival from OVCA. Acetaminophen exhibited antiproliferative activity against all tested OVCA cell lines, with half maximal inhibitory concentration values ranging from 63.2 to $403 \mu \mathrm{M}$. Pearson's correlation followed by biological pathway analysis identified 13 pathways to be associated with acetaminophen sensitivity $(\mathrm{P}<0.01)$. Associations were observed between patient survival from OVCA and expression of the following pathways: Development/angiotensin signaling via $\beta$-arrestin $(\mathrm{P}=0.04)$, protein folding and maturation/angiotensin system maturation $(\mathrm{P}=0.02)$, signal transduction/c-Jun $\mathrm{N}$-terminal kinase (JNK) pathway $(\mathrm{P}=0.03)$ and androstenedione and testosterone biosynthesis and metabolism $(\mathrm{P}=0.02)$. We confirmed that acetaminophen was active against OVCA cells in vitro. Furthermore, we identified 4 molecular signaling pathways associated with acetaminophen response that may
\end{abstract}

Correspondence to: Dr Johnathan M. Lancaster, Department of Women's Oncology, H. Lee Moffitt Cancer Center and Research Institute, 12902 Magnolia Drive, Tampa, FL 33612, USA

E-mail: Jlancaster@myriad.com

Key words: ovarian cancer, acetaminophen, paracetamol, c-Jun $\mathrm{N}$-terminal kinase pathway, molecular signaling pathways also affect overall survival in women with OVCA, including the JNK pathway, which has been previously implicated in the mechanism of action of acetaminophen and is predictive of decreased survival in women with OVCA.

\section{Introduction}

Ovarian cancer (OVCA) is the most lethal gynecological cancer, with an estimated 21,980 new cases and 14,270 deaths in 2014 (1). Unfortunately, 75\% of cases are diagnosed at an advanced (III-IV) stage due to the lack of symptomatic early-stage disease or effective screening tests. Although $\leq 70 \%$ of these women demonstrate a complete response to primary treatment with cytoreductive surgery and adjuvant platinum/taxane-based chemotherapy, the majority will eventually develop recurrent disease that is associated with poor survival $(2,3)$. Such patients frequently receive multiple lines of systemic cytotoxic therapy that may be associated with significant toxicity and detriment to the quality of life. The search for additional tolerable but efficacious drugs against OVCA has progressed slowly.

Acetaminophen is a commonly used analgesic with minimal side effects that may be active against OVCA cells (4). Acetaminophen was first synthesized in 1877 by von Mehring at Johns Hopkins University, but it was not extensively used until 1949; it was briefly recalled in 1951 due to reports of blood dyscrasias, but has been marketed since 1955 as an over-the-counter drug (5). Acetaminophen is currently a commonly used analgesic and antipyretic. Adverse events associated with acetaminophen are rare when used at therapeutic dosage; however, overdose may cause fatal hepatotoxicity. Acetaminophen is generally categorized with aspirin and aspirin-like drugs, which are known to act by inhibiting prostaglandin synthesis from arachidonic acid or other fatty acid precursors (6). While the exact mechanism of action for acetaminophen remains controversial, the prevailing hypothesis is that acetaminophen inhibits the synthesis of prostaglandins by competing at the active site of the cyclooxygenase enzyme with arachidonic acid (7).

The epidemiological reports on the effect of acetaminophen on OVCA risk and behavior are conflicting. Moysich et al (8) 
reported that regular acetaminophen use reduced the risk of OVCA [odds ratio $(\mathrm{OR})=0.56 ; 95 \%$ confidence interval (CI): 0.34-0.86]. Rodriguez-Burford et al (9) were able to demonstrate a decrease in viable OVCA cells with acetaminophen treatment compared with controls. Cramer et al (10) reported a statistically significant inverse association between the use of paracetamol, which is the European name for acetaminophen, and OVCA risk. A more recent Danish case-control study found paracetamol use to be associated with a reduced OR for developing OVCA (OR=0.82, 95\% CI: 0.74-0.92; $\mathrm{P}<0.001)$ (11). Others, however, have failed to demonstrate such an association $(12,13)$. These inconsistent findings prompted us to investigate: i) The effect of acetaminophen on OVCA cell lines; ii) the molecular signaling pathways associated with acetaminophen response; and iii) in an effort to evaluate the clinical significance of our findings, the associations of these pathways with overall patient survival from OVCA.

\section{Materials and methods}

Cell line processing. A total of 16 OVCA cell lines were subjected to treatment with acetaminophen in increasing concentrations $(0-10 \mathrm{mM})$, and the data were used to determine half maximal inhibitory concentration $\left(\mathrm{IC}_{50}\right)$ values. Prior to any treatment, the 16 cell lines also underwent Affymetrix-based analysis of baseline genome-wide expression. The Pearson's correlation test was used to identify genes that were associated with acetaminophen sensitivity, which were then analyzed for representation of molecular pathways. The genes in the top 2 pathways underwent principal component analysis (PCA) and were assigned a first principal component (PC1) score, which functions as a summary measure of pathway expression. A total of 4 clinico-genomic datasets were then queried using the PC1 scores to assess the associations between acetaminophen response pathways and patient survival from OVCA.

Cell culture. The OVCA cell lines OV90, OVCAR3, SK-OV-3 and TOV112D were obtained from the American Type Culture Collection (Manassas, VA, USA); A2780S was obtained from the European Collection of Cell Cultures (Salisbury, UK); FUOV1, IMCC3, IMCC5, IGR-OV1, MCAS, OVCA420, TOV-21-G, OVCAR2, OVCAR5, PEO1 and PEO4 were kind gifts from Dr Patricia Kruk (Department of Pathology, College of Medicine, University of South Florida, Tampa, FL, USA) and Dr Susan Murphy (Department of Obstetrics and Gynecology/Division of Gynecologic Oncology, Duke University, Durham, NC, USA). The cell lines were maintained in RPMI-1640 medium (Invitrogen, Carlsbad, CA, USA) supplemented with $10 \%$ fetal bovine serum (FBS; Fisher Scientific, Pittsburgh, PA, USA), 1\% sodium pyruvate, $1 \%$ penicillin/streptomycin (Cellgro, Manassas, VA, USA), and $1 \%$ nonessential amino acids (HyClone, Hudson, NH, USA). The cells were genotyped by short tandem repeat profiling to confirm the tissue of origin, and mycoplasma testing was performed every 6 months, in accordance with the manufacturer's protocol (Lonza, Rockland, ME, USA).

RNA extraction and microarray expression analysis. OVCA cell RNA was extracted at baseline using the RNeasy mini-kit following the manufacturer's recommendations (Qiagen, Valencia, CA, USA). The RNA quality was evaluated using an Agilent 2100 Bioanalyzer (Agilent Technologies, Inc., Santa Clara, CA, USA). The targets for Affymetrix microarray analysis were prepared according to the manufacturer's instructions and hybridized to customized Human Affymetrix HuRSTA gene chips (HuRSTA-2a520709), which include 60,607 probe sets and representation of 19,308 genes (Gene Expression Omnibus accession no. GSE34615).

CellTiter-Blue cell viability assays. Drug activity was evaluated using a high-throughput CellTiter-Blue cell viability assay. Cells $\left(2.5 \times 10^{3}\right.$ per well) were plated in 384-well plates using complete media with $10 \%$ FBS and allowed to adhere overnight. After cell adherence, increasing concentrations of acetaminophen were added to appropriate wells using an automated pipetting station. Four replicate wells were used for each drug concentration and for vehicle controls. Drug dilutions initially consisted of serial dilutions from a maximum concentration of $100 \mathrm{mM}$. The cells were incubated with the drug for $72 \mathrm{~h}$, and $5 \mu \mathrm{l}$ of CellTiter-Blue reagent (Promega Corp., Madison, WI, USA) were added to each well. Fluorescence was read at 579-nm excitation/584-nm emission using a Synergy 4 microplate reader (Bio-Tek Instruments, Inc., Winooski, VT, USA). $\mathrm{IC}_{50}$ values were determined using a sigmoidal equilibrium model fit (XLfit 5.2; ID Business Solutions Ltd., Alameda, CA, USA). The $\mathrm{IC}_{50}$ was defined as the concentration of drug required for a $50 \%$ reduction in cell growth/viability.

Statistical analysis. Expression data from the OVCA cell lines were subjected to background correction and normalization using the Robust Multichip Average algorithm in the Affymetrix Expression Console (http://www.affymetrix.com) and expressed as $\log _{2}$ values. Individual gene expression and $\mathrm{IC}_{50}$ results were evaluated using Pearson's correlation test. Probe sets demonstrating $\mathrm{P}<0.01$ were considered to be significantly correlated with the $\mathrm{IC}_{50}$ results and were analyzed using GeneGo MetaCore ${ }^{\mathrm{TM}}$ for pathway analysis (http://www.genego. com/metacore.php). Pathways demonstrating $\mathrm{P}<0.05$ (with a $30 \%$ false discovery rate) were considered significant, based on the GeneGo MetaCore ${ }^{\mathrm{TM}}$ statistical test for significance.

Clinical significance of pathways. Pathways found to be associated with acetaminophen sensitivity were tested for associations with overall survival using PCA modeling, as previously described $(14,15)$. Survival associations were evaluated by the long-rank test using median PCA value as a cut-off in 4 publically available clinico-genomic datasets from 820 women with OVCA for whom gene expression and overall survival data were available, including i) the Australian dataset ( $n=218$, GSE9891) (16), ii) the MD Anderson dataset ( $\mathrm{n}=53$, GSE18520), iii) the Cancer Genome Atlas $(\mathrm{n}=492)$, and iv) the Total Cancer Care $\left(\right.$ TCC $\left.^{\circledR}\right)$ dataset $(n=57)$.

\section{Results}

Genes and pathways associated with acetaminophen sensitivity in OVCA cell lines. Acetaminophen exhibited antiproliferative activity against all tested OVCA cell lines, 

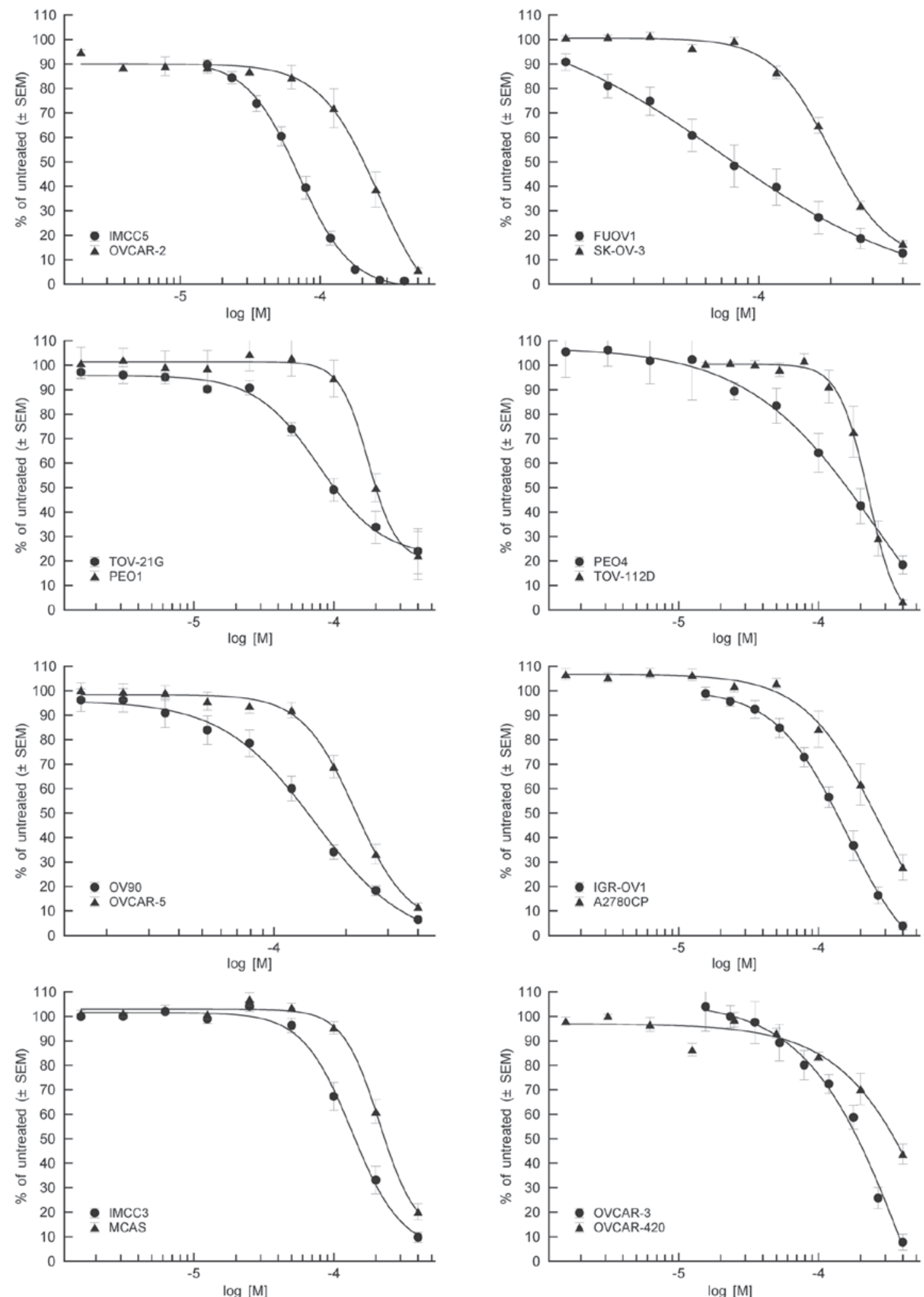

Figure 1. Line graphs showing acetaminophen dose-response curves for 16 ovarian cancer (OVCA) cell lines (percent survival vs. log acetaminophen dose). SEM, standard error of the mean.

with $\mathrm{IC}_{50}$ values ranging from 63.2 to $403 \mu \mathrm{M}$ (Fig. 1). The Pearson's correlation test using acetaminophen $\mathrm{IC}_{50}$ and $\mathrm{OVCA}$ cell line genome-wide expression data revealed the expression of 727 unique genes $(\mathrm{P}<0.01)$ representing 13 molecular signaling pathways $(\mathrm{P}<0.01)$ identified by GeneGo MetaCore ${ }^{\mathrm{TM}}$ analysis (Table I).
Pathways associated with acetaminophen sensitivity affect overall survival. PCA modeling of the 13 pathways associated with in vitro acetaminophen sensitivity in 4 publically available clinico-genomic datasets identified 4 pathways with expression that may affect overall survival from OVCA $(\mathrm{P}<0.05)$ : Development/angiotensin signaling via $\beta$-arrestin 
Table I. Biological pathways associated with acetaminophen sensitivity in 16 OVCA cell lines.

\begin{tabular}{|c|c|c|c|}
\hline Pathway name & P-value & FDR & Pathway objects \\
\hline $\begin{array}{l}\text { Protein folding and maturation/angiotensin system } \\
\text { maturation/human version }\end{array}$ & $1.13 \mathrm{E}-05$ & $6.17 \mathrm{E}-03$ & $8 / 43$ \\
\hline Cytoskeleton remodeling/neurofilaments & $3.76 \mathrm{E}-04$ & $6.86 \mathrm{E}-02$ & $5 / 25$ \\
\hline Signal transduction/JNK pathway & $6.55 \mathrm{E}-04$ & 8.96E-02 & $6 / 42$ \\
\hline Immune response/MIF - the neuroendocrine-macrophage connector & $1.07 \mathrm{E}-03$ & $1.18 \mathrm{E}-01$ & $6 / 46$ \\
\hline Androstenedione and testosterone biosynthesis and metabolism p.2 & $1.86 \mathrm{E}-03$ & 1.29E-01 & $5 / 35$ \\
\hline Cytoskeleton remodeling/keratin filaments & $2.12 \mathrm{E}-03$ & $1.29 \mathrm{E}-01$ & $5 / 36$ \\
\hline $\begin{array}{l}\text { Effect of low doses of arsenite on glucose-stimulated } \\
\text { insulin secretion in pancreatic cells }\end{array}$ & $2.12 \mathrm{E}-03$ & $1.29 \mathrm{E}-01$ & $5 / 36$ \\
\hline Development/angiotensin signaling via $\beta$-arrestin & $3.53 \mathrm{E}-03$ & $1.86 \mathrm{E}-01$ & $4 / 25$ \\
\hline Androstenedione and testosterone biosynthesis and metabolism p.3 & $3.80 \mathrm{E}-03$ & $1.86 \mathrm{E}-01$ & $5 / 41$ \\
\hline $\begin{array}{l}\text { Neurophysiological process/dopamine D2 receptor transactivation } \\
\text { of PDGFR in CNS }\end{array}$ & 4.09E-03 & $1.86 \mathrm{E}-01$ & $4 / 26$ \\
\hline Cell adhesion/gap junctions & $6.91 \mathrm{E}-03$ & 2.70E-01 & $4 / 30$ \\
\hline $\begin{array}{l}\text { Regulation of lipid metabolism/FXR-dependent negative } \\
\text { feedback regulation of bile acid concentration }\end{array}$ & $7.78 \mathrm{E}-03$ & 2.84E-01 & $4 / 31$ \\
\hline Cell cycle/role of Nek in cell cycle regulation & $8.72 \mathrm{E}-03$ & 2.98E-01 & $4 / 32$ \\
\hline
\end{tabular}

OVCA, ovarian cancer; FDR, false discovery rate; JNK; c-Jun N-terminal kinase; MIF, macrophage migration inhibitory factor; p.2, pathway 2; p.3, pathway 3; PDGFR, platelet-derived growth factor receptor; CNS, central nervous system; FXR, farnesoid X receptor; Nek, never-in-mitosis A-related kinase.

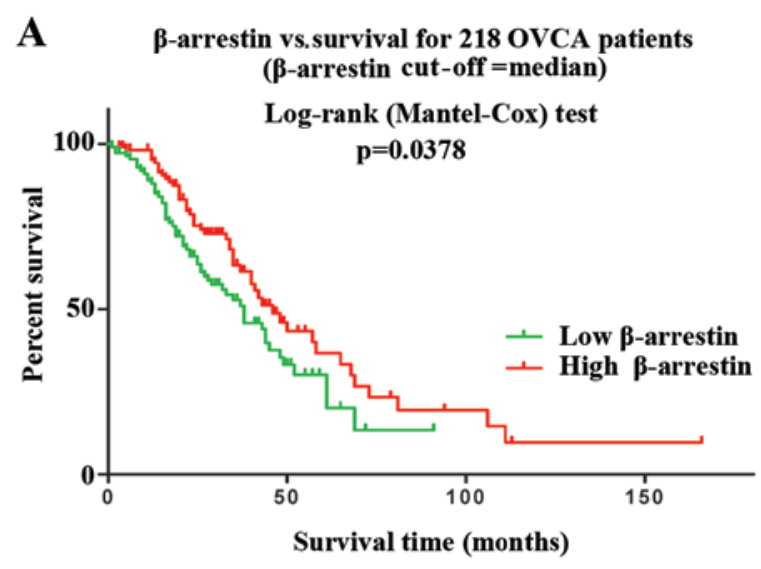

C Angiotensin vs. survival for 218 ovCA patients

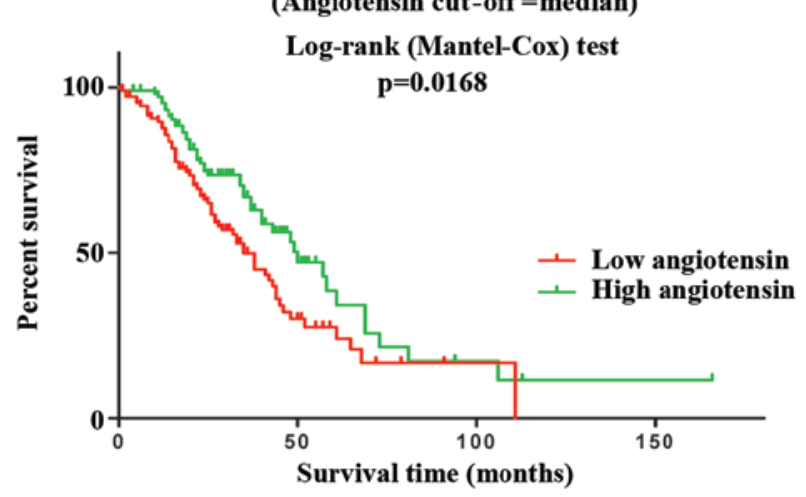

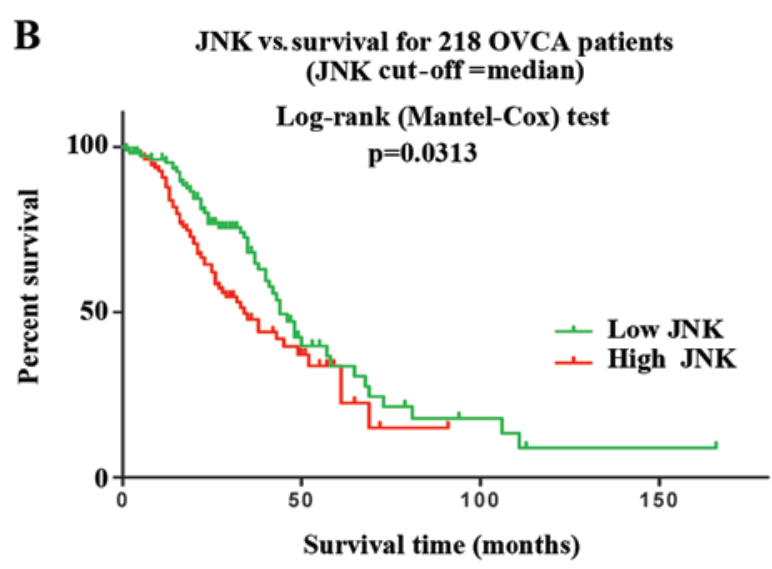

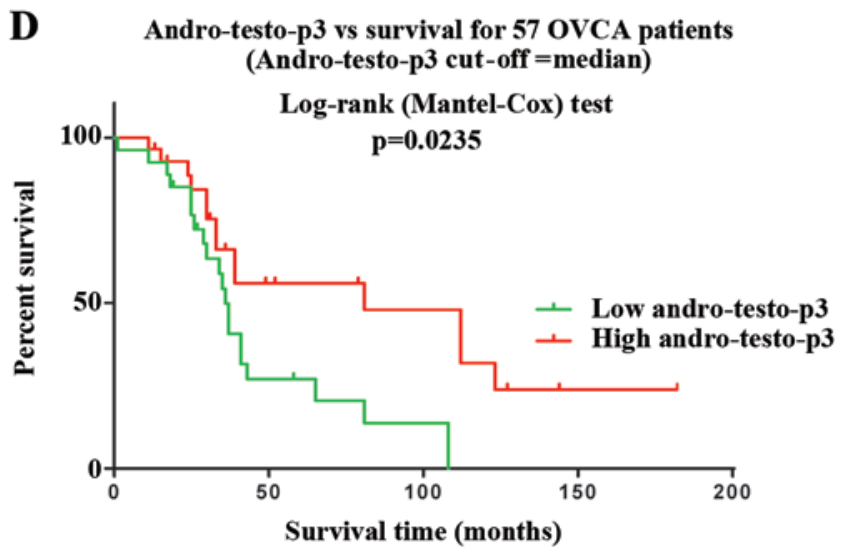

Figure 2. (A) Expression of the development/angiotensin signaling via $\beta$-arrestin; (B) signal transduction/JNK pathway; (C) protein folding and maturation - angiotensin system maturation; and (D) androstenedione and testosterone biosynthesis and metabolism pathways is associated with overall survival from OVCA. Kaplan-Meier curves depict the association between the pathway principal component analysis (PCA) score (using median PCA threshold) and overall survival in OVCA datasets. OVCA, ovarian cancer; JNK, c-Jun N-terminal kinase. 
( $\mathrm{P}=0.04$, Australian dataset) (Fig. 2A), protein folding and maturation/angiotensin system maturation $(\mathrm{P}=0.02$, Australian dataset) (Fig. 2B), signal transduction/c-Jun N-terminal kinase (JNK) pathway ( $\mathrm{P}=0.03$, Australian dataset) (Fig. 2C), and androstenedione and testosterone biosynthesis and metabolism ( $\mathrm{P}=0.02$, TCC dataset) (Fig. 2D).

\section{Discussion}

There are conflicting epidemiological and in vitro data on the effects of acetaminophen on OVCA. In this study, we demonstrated that acetaminophen exerts a measurable effect on OVCA cells and identified possible molecular determinants of OVCA sensitivity to acetaminophen. In addition, we demonstrated that overall patient survival is associated with the expression of 4 acetaminophen response molecular signaling pathways. This finding suggests a clinical relevance of the identified molecular signaling pathways in OVCA.

Acetaminophen hepatotoxicity is known to be mediated through the JNK pathway (17). Antineoplastic properties have been demonstrated in vitro, where acetaminophen has been shown to enhance the apoptotic effect of cisplatin and paclitaxel in SKOV3 human ovarian carcinoma cells through modulation of intracellular glutathione concentration (4). The epidemiological data vary widely regarding the protective effect of regular acetaminophen use against OVCA development (8-13).

Our study identified an antiproliferative effect of acetaminophen and 4 clinically relevant molecular signaling pathways that are associated with acetaminophen sensitivity in vitro. The Signal transduction/JNK, development/angiotensin signaling via $\beta$-arrestin, protein folding and maturation/angiotensin system maturation, and androstenedione and testosterone biosynthesis and metabolism pathways were significantly associated with in vitro sensitivity of OVCA cell lines to acetaminophen, and clinically relevant given their association with overall survival.

JNKs are serine/threonine kinases that belong to the mitogen-activated protein kinase (MAPK) family (18). Phosphorylation of JNK by MAPK kinase 4/7 activates JNK, which may then regulate a number of metabolic and survival pathways, as well as mediate cell death $(18,19)$. Sustained JNK activation imparts an increase in apoptosis and necrotic cell death pathways, while transient JNK activation protects against cell death (20-22). However, the presence of JNK is required for development of gastric cancer, suggesting its role in carcinogenesis (23). The JNK pathway has previously been implicated in acetaminophen-induced hepatotoxicity (17). JNK expression has been shown in a substantial number of OVCA patients and its expression was correlated to progression-free survival, while inhibition of JNK resulted in decreased tumor growth in vivo (24). While sustained activation of JNK leads to an increase in apoptosis of OVCA cells, JNK has been shown to be important in the mechanism of action of acetaminophen and has also been shown to affect OVCA survival and response to therapy (25).

We identified a potential novel implication of the development/angiotensin signaling via $\beta$-arrestin, protein folding and maturation/angiotensin system maturation, and androstenedione and testosterone biosynthesis and metabolism pathways. These three pathways have not been previously implicated in OVCA or the mechanism of action of acetaminophen; further investigation is required on the interplay of these biological processes in ovarian carcinogenesis.

In summary, we demonstrated that acetaminophen exerts antiproliferative effects on OVCA cells in vitro. By using an integrated methodology, we identified 4 molecular signaling pathways that appear to be clinically relevant for OVCA. One of these pathways (signal transduction/JNK pathway) was previously found to be involved in the mechanism of action of acetaminophen and to be predictive of decreased survival from OVCA.

\section{Acknowledgements}

We would like to thank Rasa Hamilton (H. Lee Moffitt Cancer Center and Research Institute) for editorial assistance. We would like to thank The Foundation of Women's Cancer and Moffitt's Total Cancer Care ${ }^{\circledR}$ Protocol (TCC). TCC is enabled, in part, by the generous support of the DeBartolo Family. Our study also received valuable assistance from the Translation Research Core and Cancer Informatics Core at H. Lee Moffitt Cancer Center and Research Institute; a National Cancer Institute-designated Comprehensive Cancer Center, supported under NIH grant P30 CA76292.

\section{References}

1. Siegel R, Ma J, Zou Z and Jemal A: Cancer statistics, 2014. CA Cancer J Clin 64: 9-29, 2014.

2. Ozols RF, Bundy BN, Greer BE, Fowler JM, Clarke-Pearson D, Burger RA, Mannel RS, DeGeest K, Hartenbach EM and Baergen R; Gynecologic Oncology Group: Phase III trial of carboplatin and paclitaxel compared with cisplatin and paclitaxel in patients with optimally resected stage III ovarian cancer: A Gynecologic Oncology Group study. J Clin Oncol 21: 3194-3200, 2003.

3. McGuire WP, Hoskins WJ, Brady MF, Kucera PR, Partridge EE, Look KY, Clarke-Pearson DL and Davidson M: Cyclophosphamide and cisplatin compared with paclitaxel and cisplatin in patients with stage III and stage IV ovarian cancer. N Engl J Med 334: 1-6, 1996.

4. Wu YJ, Neuwelt AJ, Muldoon LL and Neuwelt EA: Acetaminophen enhances cisplatin- and paclitaxel-mediated cytotoxicity to SKOV3 human ovarian carcinoma. Anticancer Res 33: 2391-2400, 2013.

5. Spooner JB and Harvey JG: The history and usage of paracetamol. J Int Med Res 4 (Suppl 4): 1-6, 1976.

6. Ameer B and Greenblatt DJ: Acetaminophen. Ann Int Med 87: 202-209, 1977.

7. Botting RM: Mechanism of action of acetaminophen: Is there a cyclooxygenase 3? Clin Infect Dis 31 (Suppl 5): S202-S210, 2000.

8. Moysich KB, Mettlin C, Piver MS, Natarajan N, Menezes RJ and Swede H: Regular use of analgesic drugs and ovarian cancer risk. Cancer Epidemiol Biomarkers Prev 10: 903-906, 2001.

9. Rodriguez-Burford C, Barnes MN, Oelschlager DK, Myers RB, Talley LI, Partridge EE and Grizzle WE: Effects of nonsteroidal anti-inflammatory agents (NSAIDs) on ovarian carcinoma cell lines: Preclinical evaluation of NSAIDs as chemopreventive agents. Clin Cancer Res 8: 202-209, 2002.

10. Cramer DW, Harlow BL, Titus-Ernstoff L, Bohlke K, Welch WR and Greenberg ER: Over-the-counter analgesics and risk of ovarian cancer. Lancet 351: 104-107, 1998.

11. Baandrup L, Friis S, Dehlendorff C, Andersen KK, Olsen JH and Kjaer SK: Prescription use of paracetamol and risk for ovarian cancer in Denmark. J Natl Cancer Inst 106: dju111, 2014.

12. Lo-Ciganic WH, Zgibor JC, Bunker CH, Moysich KB, Edwards RP and Ness RB: Aspirin, nonaspirin nonsteroidal anti-inflammatory drugs, or acetaminophen and risk of ovarian cancer. Epidemiology 23: 311-319, 2012. 
13. Hannibal CG, Rossing MA, Wicklund KG and Cushing-Haugen KL: Analgesic drug use and risk of epithelial ovarian cancer. Am J Epidemiol 167: 1430-1437, 2008.

14. Marchion DC, Cottrill HM, Xiong Y, Chen N, Bicaku E, Fulp WJ, Bansal N, Chon HS, Stickles XB, Kamath SG, et al: BAD phosphorylation determines ovarian cancer chemosensitivity and patient survival. Clin Cancer Res 17: 6356-6366, 2011.

15. Chen DT, Nasir A, Culhane A, Venkataramu C, Fulp W, Rubio R, Wang T, Agrawal D, McCarthy SM, Gruidl M, et al: Proliferative genes dominate malignancy-risk gene signature in histologically-normal breast tissue. Breast Cancer Res Treatment 119: 335-346, 2010.

16. Tothill RW, Tinker AV, George J, Brown R, Fox SB, Lade S, Johnson DS, Trivett MK, Etemadmoghadam D, Locandro B, et al: Novel molecular subtypes of serous and endometrioid ovarian cancer linked to clinical outcome. Clin Cancer Res 14: 5198-5208, 2008.

17. Win S, Than TA, Han D, Petrovic LM and Kaplowitz N: c-Jun $\mathrm{N}$-terminal kinase (JNK)-dependent acute liver injury from acetaminophen or tumor necrosis factor (TNF) requires mitochondrial Sab protein expression in mice. J Biol Chem 286: 35071-35078, 2011.

18. Johnson GL and Nakamura K: The c-jun kinase/stress-activated pathway: Regulation, function and role in human disease. Biochim Biophys Acta 1773: 1341-1348, 2007.
19. Weston CR and Davis RJ: The JNK signal transduction pathway. Curr Opin Cell Biol 19: 142-149, 2007.

20. Czaja MJ: Cell signaling in oxidative stress-induced liver injury. Semin Liver Dis 27: 378-389, 2007.

21. Han D, Ybanez MD, Ahmadi S, Yeh K and Kaplowitz N: Redox regulation of tumor necrosis factor signaling. Antioxidants Redox Signal 11: 2245-2263, 2009.

22. Gunawan BK, Liu ZX, Han D, Hanawa N, Gaarde WA and Kaplowitz N: c-Jun N-terminal kinase plays a major role in murine acetaminophen hepatotoxicity. Gastroenterology 131: 165-178, 2006.

23. Shibata W, Maeda S, Hikiba Y, Yanai A, Sakamoto K, Nakagawa H, Ogura K, Karin M and Omata M: c-Jun $\mathrm{NH} 2$-terminal kinase 1 is a critical regulator for the development of gastric cancer in mice. Cancer Res 68: 5031-5039, 2008.

24. Vivas-Mejia P, Benito JM, Fernandez A, Han HD, Mangala L, Rodriguez-Aguayo C, Chavez-Reyes A, Lin YG, Carey MS, Nick AM, et al: c-Jun- $\mathrm{NH}_{2}$-kinase-1 inhibition leads to antitumor activity in ovarian cancer. Clin Cancer Res 16: 184-194, 2010.

25. Mansouri A, Ridgway LD, Korapati AL, Zhang Q, Tian L, Wang Y, Siddik ZH, Mills GB and Claret FX: Sustained activation of JNK/p38 MAPK pathways in response to cisplatin leads to Fas ligand induction and cell death in ovarian carcinoma cells. J Biol Chem 278: 19245-19256, 2003. 